[0212-7199 (2007) 24: 6; pp 278-280] ANALES DE MEDICINA INTERNA Copyright (C) 2007 ARAN EDICIONES, S.L

AN. MED. INTERNA (Madrid) Vol. 24, N. $^{\circ} 6$, pp. $278-280,2007$

\title{
¿Es la tomografía por emisión de positrones útil en el estudio de extensión de los tumores GIST?
}

\author{
M. J. MOLINA GARRIDO, C. GUILLÉN PONCE \\ Servicio de Oncología Médica. Hospital General Universitario de Elche. Alicante
}

IS POSITRON EMISSION TOMOGRAPHY (PET) SCAN NECESSARY IN GASTROINTESTINAL STROMAL TUMORS?

\begin{abstract}
RESUMEN
Los tumores gastrointestinales (GIST) son los tumores malignos mesenquimales más frecuentes del tracto gastrointestinal. El hígado y el peritoneo son las localizaciones más frecuentes de las metástasis. La cirugía ha resultado ser el tratamiento de elección de estos tumores, y, cuando son metastásicos o irresecables, Imatinib se ha convertido en la terapia más eficaz de la que se dispone.

Presentamos el caso de una paciente diagnosticada de un GIST ileal, cuyo estudio de extensión fue negativo, salvo por la tomografía por emisión de positrones (PET), que indicaba que existía una lesión residual a nivel de fosa iliaca derecha. Tras el tratamiento con Imatinib, 400 $\mathrm{mg} /$ día, se consiguió una respuesta completa de la enfermedad, también reflejada en la PET.
\end{abstract}

PALABRAS CLAVE: Tomografía por emisión de positrones. Tumores gastrointestinales. Imatinib.

\begin{abstract}
Gastrointestinal tumor (GIST) is the most frequent mesenchymal malignancy of the gastrointestinal tract. Liver and peritoneum are the most frequent metastatic sites. Surgery is the standard treatment in patients with localized disease and imatinib mesylate has been shown to be an effective treatment in metastatic or unresectable disease.

We present a case of ileal GIST that was diagnosed as metastatic just with the findings of the fluorodeoxyglucose positron emission tomography (PET) and showed a total response with Imatinib on PET.
\end{abstract}

KEY WORDS: Fluorodeoxyglcose positron emission tomography. Gastrointestinal tumors. Imatinib.

Molina Garrido MJ, Guillén Ponce C. ¿Es la tomografía por emisión de positrones útil en el estudio de extensión de los tumores GIST? . An Med Interna (Madrid) 2007; 24: 278-280.

\section{INTRODUCCIÓN}

Los tumores gastrointestinales (GIST) son los tumores malignos mesenquimales más frecuentes del tracto gastrointestinal. El hígado y el peritoneo son las localizaciones más frecuentes de las metástasis. La cirugía ha resultado ser el tratamiento de elección de estos tumores, y, cuando son metastásicos o irresecables, Imatinib se ha convertido en la terapia más eficaz de la que se dispone.

\section{CASO APORTADO}

Paciente de 72 años de edad con antecedentes de ulcus gástrico, diabetes mellitus tipo 2 insulinizada, angina estable, hipertensión arterial, dislipemia y accidente cerebrovascular isquémico con secue- la residual en mano derecha. Tratamiento habitual con clopidogrel, carvedilol, nitroglicerina transdérmica, atorvastatina y omeprazol.

Consulta en febrero 2005 por masa a nivel de fosa iliaca derecha. En exploración ginecológica, presenta una lesión que parece depender de útero. En marzo 2005, se le practica histerectomía y doble anexectomía, junto con resección del tercio final de íleon, al identificarse una masa fibrosada adherida a éste. Anatomía patológica: endometrio atrófico, cervicitis crónica folicular; tumor gastrointestinal del estroma (GIST) ileal de $8 \mathrm{~cm}$ de tamaño, con 2-3 metástasis por cada 50 campos de gran aumento, hipercelularidad, celularidad epitelioide, pleomorfismo celular, necrosis focal; c-kit positivo. Valorada en consultas externas de Oncología Médica, se solicita como estudio de extensión: analítica completa: hemoglobina 11,7 $\mathrm{mg} / \mathrm{dl}$, con resto de parámetros normales; tomografía axial computerizada (TAC) toraco-abdomino-pélvica: colelitiasis, pequeño nódulo suprarrenal derecho de $2 \mathrm{~cm}$ de características benignas; gammagra-

Trabajo aceptado: 9 de enero de 2007

Correspondencia: María José Molina Garrido. Hospital General Universitario de Elche. Camí de l'Almazara, 11. 03232 Elche. Alicante. e-mail: mjmolinagarrido@hotmail.com 


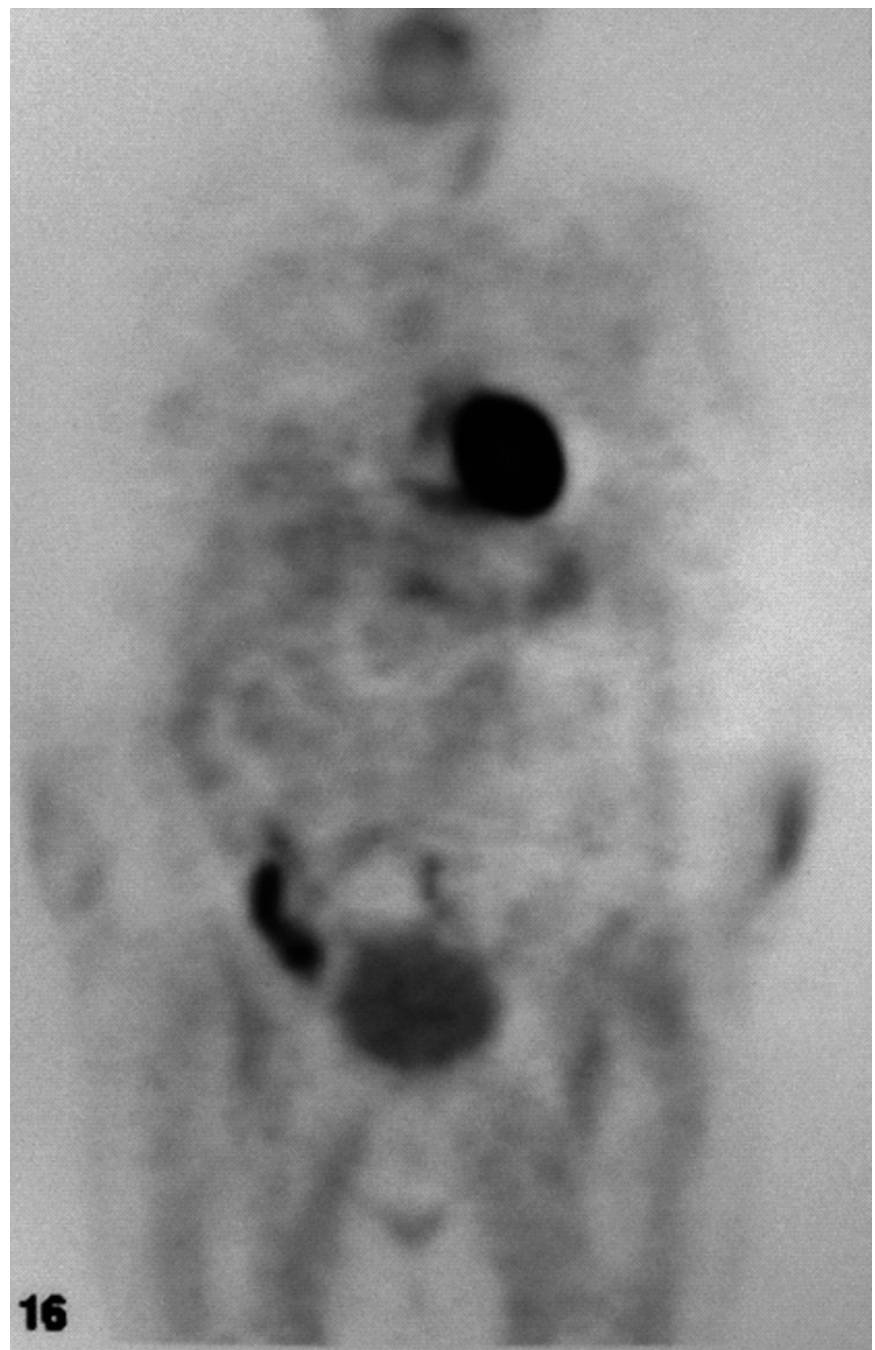

Fig. 1. PET tras la cirugía.

fía ósea: negativa para metástasis óseas. En octubre se solicitó una tomografía por emisión de positrones (PET), que reflejó la presencia de un depósito patológico de fluorodeoxiglucosa (FDG) a nivel de fosa iliaca derecha, localizado sobre asas intestinales, con un SUV máximo de 8,12 (Fig. 1). Ante estos hallazgos, se inicia tratamiento con imatinib a dosis de $400 \mathrm{mg} / \mathrm{día}$. La tolerancia a dicho tratamiento ha sido adecuada. En el nuevo estudio de reevaluación, tres meses tras el inicio del tratamiento, persiste el nódulo suprarrenal derecho, sin cambios en el tamaño ni en el aspecto, y la PET no revela la presencia de ninguna lesión residual (Fig. 2).

\section{DISCUSIÓN}

Los tumores del estroma gastrointestinal (GIST) constituyen un grupo de tumores mesenquimatosos que se desarrollan principalmente en el tracto gastrointestinal (estómago $>50 \%$; intestino delgado 20-30\%; intestino grueso $10 \%$; esófago 2$3 \%$; resto de la cavidad abdominal 5\%), aunque también existe localizaciones extragastrointestinales. Representan menos del $1 \%$ de todos los tumores gastrointestinales (1).

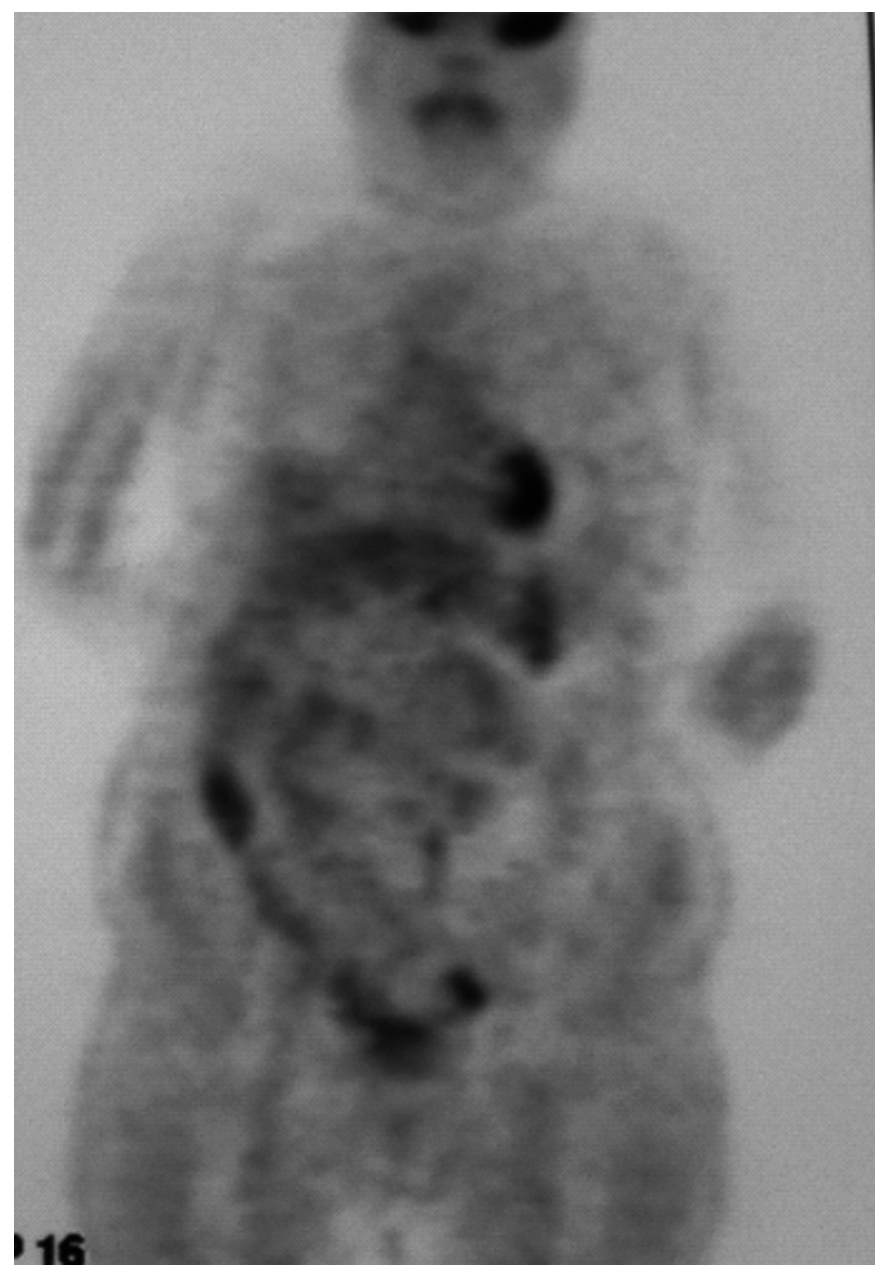

Fig. 2. PET tras tres meses de tratamiento.

Hasta ahora, la resección quirúrgica agresiva ha sido el tratamiento de elección (cirugía con bordes amplios, sin linfadenectomía), porque la quimioterapia estándar y la radioterapia no han demostrado ningún beneficio, con una supervivencia global a los 5 años del $35 \%$ (2).

Las manifestaciones clínicas de los GIST dependen de su localización. En este caso, la paciente permaneció asintomática en todo momento, salvo por la existencia de una masa abdominal, cuyo estudio ginecológico indicaba que podría tratarse de una tumoración uterina.

Una característica imprescindible para llegar a su diagnóstico, es su intensa positividad frente al KIT (CD117), que se detecta en el 90-100\% de las células tumorales, dato que estaba presente en nuestro caso. Basándose en este hecho, se ha elaborado un fármaco, un inhibidor de la tirosin-kinasa, el STI571 (Imatinib), que proporciona, al menos, respuestas parciales en casi el $69 \%$ de los pacientes con un GIST metastásico (3). Incluso en tumores de bajo riesgo, el $50 \%$ recidiva dentro de los 5 primeros años de seguimiento (4), y en el momento de presentación, entre el 15 y el $50 \%$ de los GIST son metastásicos (hígado o peritoneal). El tamaño y la extensión de los GIST se determina normalmente mediante TAC (5). 
La tomografía por emisión de positrones (PET) se ha empleado en los tumores GIST con distintas finalidades. Es un método muy útil para la detección de una respuesta temprana (1 semana tras iniciar el tratamiento) a Imatinib en pacientes con tumores GIST $c$-kit positivos (6). Hay estudios que muestran que la PET es capaz de detectar la reducción en el tamaño de las metástasis, incluso 2,8 días antes que los hallazgos de la TAC (7). Incluso la PET parece predecir el grado de malignidad de los tumores GIST gástricos antes de la intervención quirúrgica, basándose en la intensidad de la captación (8). También es útil en el seguimiento de los pacientes tratados con Imatinib, y permite distinguir entre los efectos secundarios del tratamiento y la progresión tumoral (9).

Sin embargo, hasta el momento, con los datos disponibles en la literatura, no se ha descrito ningún caso de tumor GIST en el que las metástasis se hubieran detectado sólo en la PET. En nuestro caso, fue gracias a esta técnica de imagen como se consiguió detectar que se trataba de una enfermedad metastásica, lo que permitió el inicio de tratamiento con Imatinib (no está aprobado su uso generalizado en adyuvancia de los GIST). Aunque se ha descrito casos de PET positiva tras la cirugía, secundario a inflamación y fibrosis, y la negativización de la misma tras varios meses, sin la administración de ningún tratamiento, en nuestra paciente, transcurrió casi 7 meses entre la intervención quirúrgica y la realización de la primera PET, lo que hace muy improbable que se tratara de un falso positivo. A los tres meses del inicio del tratamiento, se empleó de nuevo la PET como método de reevaluación. El resultado fue la obtención de una respuesta completa.

Este caso podría servir para que se valore la utilización de la PET en el estudio inicial de extensión de todos los pacientes con tumores GIST, teniendo en cuenta que permite detectar lesiones que pasan desapercibidas al resto de pruebas de imagen.

\section{Bibliografía}

1. Pidhorecky I, Chney RT, Kraybill WG, et al. Gastrointestinal stromal tumors: Current diagnosis, biologic behaviour, and management. Ann Surg Oncol 2000; 7: 705-12.

2. DeMatteo RP, Lewis JJ, Leung D, et al. Two hundred gastrointestinal stromal tumors: Recurrence patterns and prognostic factors for survival. Ann Surg 2000; 231: 51-8.

3. van Oosterom AT, Judson I, Verweij J, et al. Safety and efficacy of imatinib (STI571) in metastatic gastrointestinal stromal tumours: A phase I study. Lancet 2001; 358: 1421-3.

4. DeMatteo RP, Lewis JJ, Leung D, et al. Two hundred gastrointestinal stromal tumors: Recurrence patterns and prognostic factors for survival. Ann Surg 2000; 231: 51-8.

5. Nishida T, Kumano S, Sugiura T, et al. Multidetector CT of high-risk patients with occult gastrointestinal stromal tumors. AJR Am J Roentgenol 2003; 180: 185-9.
6. Heinicke T, Wardelmann E, Sauerbruch T, et al. Very early detection of response to imatinib mesylate therapy of gastrointestinal stromal tumours using 18 fluoro-deoxyglucose-positron emisión tomography. Anticancer Res 2005; 25 (6C): 4591-4.

7. Goldstein D, Tan BS, Rossleigh M, et al. Gastrointestinal Stromal Tumours: Correlation of 18F-FDG Gamma Camera-based coincidence positron emission tomography with CT for the assessment of treatment response-An AGIGT Study. Oncology 2005; 69: 326-32.

8. Kamiyama Y, Aihara R, Nakabayashi T, et al. 18 F-Fluorodeoxyglucose Positron Emission Tomography: Useful technique for predicting malignant potential of gastrointestinal stromal tumors. W J Surg 2005; 29: 1429-35.

9. Jager PL, Gietema JA, van der Graaf WTA. Imatinib mesylate for the treatment of gastrointestinal stromal tumours: Best monitored with FDG PET. Nucl Med Commun 2004; 25: 433-8. 